\title{
Structure-Activity Relationships of 9-mer Antimicrobial Peptide analogue of Protaetiamycine, 9Pbw2
}

\author{
Jin-Kyoung Kim, Eunjung Lee, Ki-woong Jung, Yangmee Kim* \\ Department of Bioscience and Biotechnology, Konkuk University, Seoul 143-701, Korea \\ (Received May 07, 2011; accepted May 31, 2011)
}

\begin{abstract}
Pbw2 is a 9-mer analog of protaetiamycine derived from the larvae of the beetle Protaetia brevitarsis. Previously, we designed four 9mer peptide analogues to optimize the balance between the hydrophobicity and cationicity of the peptides and to increase bacterial cell selectivity. Among them, 9Pbw2 has high antibacterial activity without cytotoxicity. The results obtained in previous study suggest that the bactericidal action of 9Pbw2 may be attributed to the inhibition of the functions of intracellular components after penetration of the bacterial cell membrane. In order to understand structure-activity relationships, we determined the threedimensional structure of 9Pbw2 in $200 \mathrm{mM}$ DPC micelle by NMR spectroscopy. 9Pbw2 has one hydrophobic turn helix from $\operatorname{Trp}^{3}$ to $\mathrm{Arg}^{8}$ and positively charged residues at the $\mathrm{N}$ - and C-terminus. This result suggested that positively charged residues from position at the C-terminus in 9Pbw2 may be important for the primary binding to the negatively charged phospholipid head groups in bacterial cell membranes and hydrophobic residues in the middle portion face toward the acyl chains of the hydrophobic lipid in the bacterial cell membrane.
\end{abstract}

Keywords : protaetiamycine analogue, antimicrobial peptide, bacterial cell selectivity, NMR, structure

\footnotetext{
* To whom correspondence should be addressed. E-mail : ymkim@konkuk.ac.kr
} 


\section{INTRODUCTION}

Searching for novel types of antibiotics which is not been exposed against microorganisms has become more important. Among the possible candidates, antimicrobial peptides are attractive one ${ }^{1}$. Although the detailed mechanisms are not fully understood, many of these antimicrobial peptides rapidly kill invading pathogens by causing membrane permeabilization or inhibition of the functions of intracellular components after penetration. ${ }^{2-4}$ We have previously identified a novel insect defensin, protaetiamycine from the larvae of a beetle, Protaetia brevitarsis. ${ }^{5}$ Since a short antimicrobial peptide with bacterial cell selectivity would be an attractive candidate for a therapeutic agent, we designed four 9-mer analogues of protaetiamycine on the basis of its sequence from $\mathrm{Ala}^{22}$ to $\mathrm{Gly}^{30}$. Among the analogues, 9Pbw2 (RLWLAIKRR-NH $\left.{ }_{2}\right), \quad 9 P b w 3 \quad\left(\right.$ RLWLAIWRR-NH $\left.H_{2}\right), \quad$ and $9 P b w 4$ (RLWLAWKRR-NH ${ }_{2}$ ) showed high antimicrobial activities while only 9Pbw2 and 9Pbw4 are bacterial cell selective. Our previous study showed that mechanisms of action of 9Pbw2 and 9Pbw4 are related to penetration of bacterial cell membrane. ${ }^{6}$ In this study, to understand the structureactivity relationships of 9Pbw2 and interactions between 9Pbw2 and membrane, the tertiary structures of 9Pbw2 in DPC micelle has been determined using NMR spectroscopy. 


\section{EXPERIMENTAL}

\section{Sample preparation}

Peptide was prepared by solid-phase synthesis using Fmoc chemistry. Peptide was purified by reversed-phase preparative high-performance liquid chromatography on a C18 column $(20 \times 250$ mm; Shim-pack) using a gradient of $20-50 \%$ acetonitrile in $\mathrm{H}_{2} \mathrm{O}$ with $0.1 \%$ TFA delivered over 30 min. Analytical high-performance liquid chromatography with an octadecylsilica (ODS) column (4.6 × $250 \mathrm{~mm}$; Shim-pack) revealed that purified peptide was more than 95\% homogeneous (data not shown). The peptide also had the correct atomic masses as determined by matrix-assisted laser desorption/ionization time-of-flight mass spectrometry.

\section{Tryptophan Fluorescence blue shift experiment}

Intrinsic tryptophan fluorescence studies of 9Pbw2 were performed in $10 \mathrm{mM}$ Tris buffer at a peptide concentration of $5 \mu \mathrm{M}$ and at $\mathrm{pH}$ 7.4. The interaction of 9Pbw2 with DPC or SDS micelles was investigated by recording the fluorescence emission spectra of the peptide as a function of the concentration of DPC micelles compared with that in SDS micelles. 


\section{NMR spectroscopy}

9Pbw2 was dissolved at $1.0 \mathrm{mM}$ in $0.45 \mathrm{ml}$ of a 9:1 (v/v) $\mathrm{H}_{2} \mathrm{O} / \mathrm{D}_{2} \mathrm{O}(\mathrm{pH}$ 6.0) solution containing 200 mM DPC micelles. All of the NMR experiments for the sample in DPC micelle were performed at 303K. All the phase sensitive two-dimensional experiments such as DQF-COSY, TOCSY, and NOESY experiments were performed using time-proportional phase incrementation (TPPI) method. ${ }^{7-}$

${ }^{11}$ For these experiments, 400-512 transients with $2 \mathrm{~K}$ complex data points were collected for each of the increments with a relaxation delay of 1.2-1.5 sec between the successive transients and the data along the $t_{1}$ dimension were zerofilled to $1 \mathrm{~K}$ before $2 \mathrm{D}$-Fourier transformation. TOCSY experiment was performed with mixing times of 50-80 ms, MLEV-17 spin-lock mixing pulse. NOESY experiments were performed with mixing times of 250 and 350 ms. All NMR spectra were recorded on Bruker DPX-400 spectrometer in Konkuk University.

Chemical shifts of the samples were measured relative to the methyl resonance of internal 2,2dimethyl-2-silapentane-5-sulfonic acid (DSS) at 0 ppm.

\section{Structure calculation}

Structure calculations were carried out using X-PLOR version $3.851 .^{12}$ All the NOE intensities are divided into three classes, i.e., strong, medium, and weak with the distance ranges of $1.8-2.7,1.8-$ 3.5, and 1.8-5.0 $\AA$, respectively. ${ }^{13,14}$ Standard pseudoatom corrections were applied to the non- 
stereospecifically assigned restraints, and the additional $0.5 \AA$ was added to the upper bounds for NOEs involving methyl protons. ${ }^{15}$ Standard distance geometry-dynamical simulated annealing hybrid protocol $^{16,17}$ was employed to generate structures. Center averaging was used to correct distances involving methyl groups and non-stereospecifically assigned methylene. The target function that is minimized during simulated annealing comprises only quadratic harmonic potential terms for covalent geometry, square-well quadratic potentials for the experimental distance and torsion angle restraints, and a quartic van der Waals repulsion term for the nonbonded contacts.

\section{Diffusion Ordered Spectroscopy}

DOSY has been used to determine an accurate and precise value of the self-diffusion constant D. ${ }^{18}$ DOSY experiments were performed on a Bruker $500 \mathrm{MHz}$ Spectrometer equipped with a z-axis gradient coil. The gradient strength was calibrated using DPC resonance signal in water. ${ }^{1} \mathrm{H}$ detected 2D DOSY data sets were recorded using the stimulated echo sequence with bipolar-gradient pulses and $16 \mathrm{~T}_{1}$ blocks of 128 transients each. The diffusion time was $100 \mathrm{~ms}$ and duration of the gradient pulse was 1.5 ms to obtain a $2 \%$ residual signal with the maximum gradient strength. The 2D data sets were processed with the two-exponential decays using the BIOSPIN software and consisted of an exponential-multiplication apodization function $(\mathrm{lb}=1 \mathrm{~Hz})$ in the $\mathrm{F}_{2}$ dimension prior to Fourier transformation. The $T_{1}$ diffusion dimension was processed by fitting the intensity of the resultant $F_{2}$ peaks to following equation, ${ }^{18}$ 


$$
I=I_{0} \exp \left(-D \gamma^{2} g^{2} \delta^{2}(\Delta-\delta / 3-\tau / 2)\right)
$$

where $I$ is observed intensity, $I_{0}$ the reference intensity (unattenuated signal intensity), $D$ is the diffusion coefficient in $\mathrm{m}^{2} \mathrm{~s}^{-1}, \gamma$ is the ${ }^{1} \mathrm{H}$ gyromagnetic ratio $\left(4257.7002 \mathrm{~Hz} \cdot \mathrm{G}^{-1}\right), g$ is the gradient strength that consisted of 16 linear increments between 0.83 and $39.3 \mathrm{G} \mathrm{cm}^{-1}$ (2-95\% gradient strength) which accounts for the $16 \mathrm{~T}_{1}$ increments, $\delta$ is the total length of the bipolar defocusing/refocusing gradient pulses, $\Delta$ is the diffusion time, and $\tau / 2$ is the delay time between the two bipolar gradient pulses which depends on spectrometer constant.

\section{RESULTS AND DISCUSSION}

We have reported the antimicrobial activities and cytotoxicity of four 9-mer peptide analogues in the previous paper. 9Pbw2 shows high antibacterial activity without cytotoxicity, resulting in high bacterial cell selectivity. Here, fluorescence emissions of tryptophan residue in this peptide were monitored in Tris-HCl Buffer ( $\mathrm{pH}$ 7.4) or in the presence of neutral lipids, DPC micelles or in negatively charged lipids SDS micelles (Table 1). Melittin, which is known to have not only high antibacterial activity but also high cytotoxicity, showed large blue shift in both lipids, implying that melittin is not cell selective. 9Pbw2 exhibited considerably smaller blue shifts in neutral lipids DPC micelles than in the negatively charged lipids SDS micelles, resulting in high bacterial cell selectivity. 
Table 1. Tryptophan emission maximum of the peptide in Tris-HCl buffer ( $\mathrm{pH} 7.4)$ or in the presence of DPC micelles and SDS micelles

\begin{tabular}{cccc}
\hline Peptide & Tris-buffer $(\mathrm{pH} \mathrm{7.4)}$ & DPC micelles & SDS micelles \\
\hline 9Pbw2 & 350 & $350(0)$ & $336(-14)$ \\
Melittin & 350 & $342(-6)$ & $332(-16)$ \\
\hline
\end{tabular}

Using standard sequential assignment strategy, all the proton resonances were assigned. TOCSY and DQF-COSY spectra were used to assign spin systems of most of the amino acid residues. By direct comparison of TOCSY and NOESY spectra, sequence-specific resonance assignments were completed. The sequential NOE connectivities in the $\mathrm{NH}-\mathrm{C}_{\alpha} \mathrm{H}$ region of NOESY spectra of 9Pbw2 in DPC micelle are illustrated in Figure 1.

Figure 2 illustrates the summary of the NOE connectivities of 9Pbw2 in DPC micelles, which was extracted directly from NOESY spectrum recorded with a mixing time of 250 ms. A number of nonsequential NOE connectivities that are characteristics of an $\alpha$-helix, specifically $d_{\alpha \beta}(i, i+3)$ and $d_{\alpha N}(i, i+3)$, were observed for residue from $\operatorname{Trp}^{3}$ to $\operatorname{Arg}^{8}$ of the peptide. 


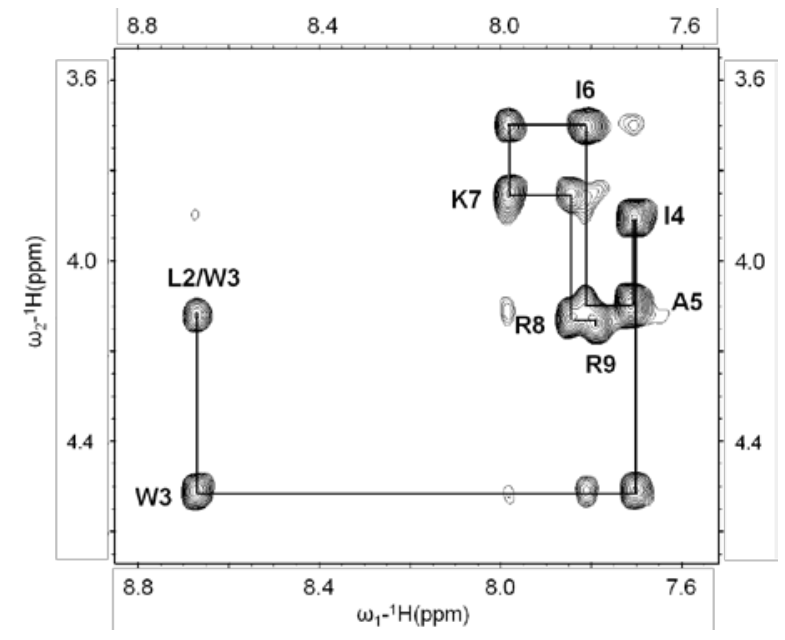

Figure 1. $\mathrm{NH}-\mathrm{C}_{\alpha} \mathrm{H}$ region of the NOESY spectra of 9Pbw2 in $200 \mathrm{mM}$ DPC micelles.

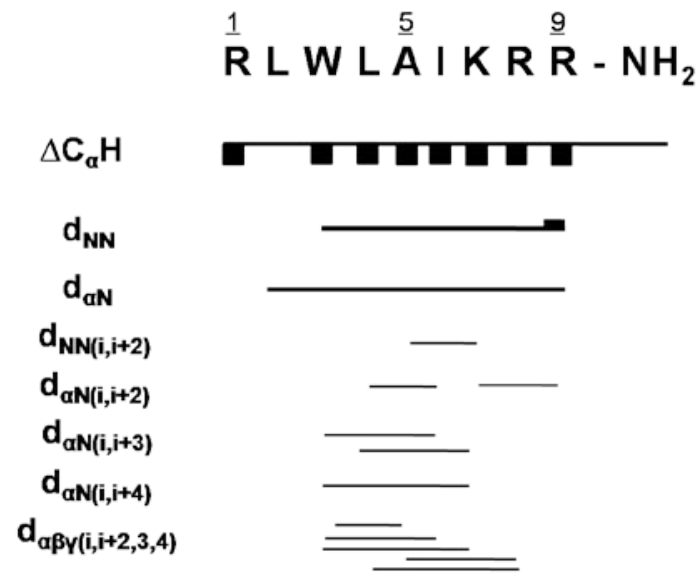

Figure. 2. Summary of the NOE connectivities and $\mathrm{C}_{\alpha} \mathrm{H}$ chemical shift indices for $9 \mathrm{Pbw} 2 \mathrm{in} 200 \mathrm{mM}$ DPC micelles. The thickness of the line for the NOEs reflects the intensity of the NOE connectivities. 
Total of 100 structures were generated by hybrid distance geometry-dynamical simulated annealing algorithm, and 20 structures having lowest energies were selected for further analysis. All of structures satisfies the experimental NOEs well within $0.02 \AA$. All structures display good covalent geometry and small NMR constraint violations. Figure 3(A) shows the superposition of the 20 lowest energy structures of 9Pbw2 over the backbone atoms in $200 \mathrm{mM}$ DPC micelles. None of the structures have violations over $0.5 \AA$ from the NOE distance restraints or 3 degrees from dihedral angle restraints, and all the structures exhibit good covalent geometry. When we superimposed the 20 lowest energy structures of 9Pbw2 over the backbone atoms (from $\operatorname{Trp}^{3}$ to $\operatorname{Arg}^{8}$ ), their root mean squared deviations from the mean structures were $0.361 \pm 0.15 \AA$ for the backbone atoms $\left(\mathrm{N}, \mathrm{C} \alpha, \mathrm{C}^{\prime}\right.$, O) and $0.973 \pm 0.23 \AA$ for all heavy atoms, respectively. As shown in figure 3 (B) and (C), 9Pbw2 has a hydrophobic one turn helix from $\operatorname{Trp}^{3}$ to $\mathrm{Arg}^{8}$ and three positively charged residues at the Cterminus. 


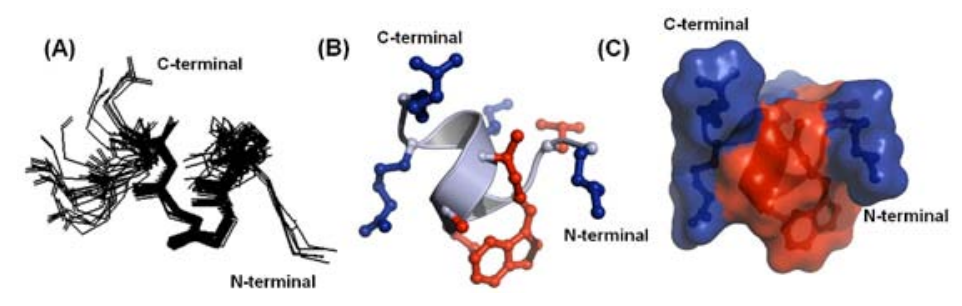

Figure. 3. (A) The superpositions of the 20 lowest energy structures calculated from the NMR data for 9Pbw2 the backbone atoms of residues $\operatorname{Trp}^{3}$ to $\mathrm{Arg}^{8}$ superimposed. (B) Ribbon diagram of the lowest energy structure (C) Surface model of 9Pbw2 in 200 mM DPC micelles. The hydrophobic side chains are indicated in red, and the hydrophilic side chains are shown in blue.
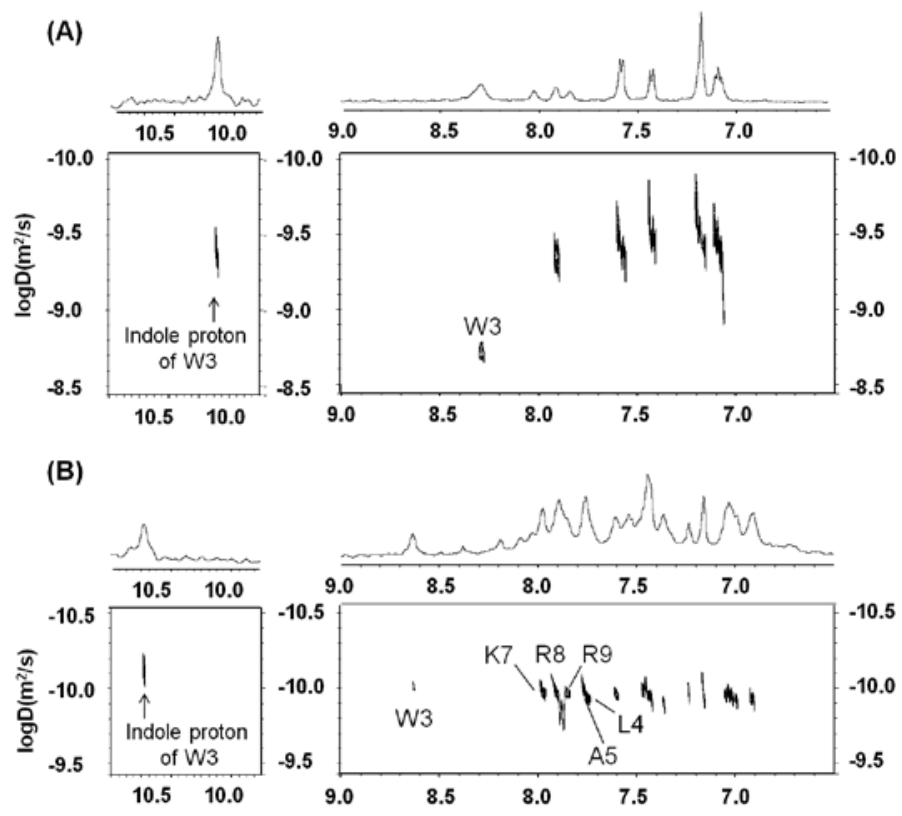

Figure. 4. The NH region of the ${ }^{1} \mathrm{H} 1 \mathrm{D}$ spectra and DOSY spectra for (A) 9Pbw2 in a 9:1 (v/v) $\mathrm{H}_{2} \mathrm{O} / \mathrm{D}_{2} \mathrm{O}$ and (B) 9Pbw2 in $200 \mathrm{mM}$ DPC micelles at 298K. 
In order to investigate the interactions between 9Pbw2 and phospholipid membrane, DOSY experiments were performed. The NH region of the 1D and DOSY spectra of the 9Pbw2 in a 9:1 (v/v) $\mathrm{H}_{2} \mathrm{O} / \mathrm{D}_{2} \mathrm{O}$ and 9Pbw2 in $200 \mathrm{mM}$ DPC micelles are shown in Figure 4. As illustrated in the spectra of 9Pbw2, addition of DPC resulted in the downfield shift of one indole proton of $\operatorname{Trp}^{3}$ in the $10 \mathrm{ppm}-11 \mathrm{ppm}$ region. Also, large chemical shift changes in the $\mathrm{NH}$ and the aromatic ring regions were seen. These changes imply that 9Pbw2 has unordered structures in aqueous solution while they form more ordered structures in micellar environments which mimic the amphipathic environment of a phospholipid bilayer. It is seen that the average value of diffusion coefficient of the peptide was decreased from $3.55 \times 10^{-10} \mathrm{~m}^{2} \mathrm{~s}^{-1}$ to $1.08 \times 10^{-10} \mathrm{~m}^{2} \mathrm{~s}^{-1}$ when bound to DPC micelles. This result suggested that 9Pbw2 interact with DPC micelles and diffuses slowly in bound form. The amide proton of $\operatorname{Trp}^{3}$ shows dramatic changes of diffusion coefficients from $1.92 \times 10^{-9} \mathrm{~m}^{2} \mathrm{~s}^{-1}$ for free form to $9.62 \times 10^{-11} \mathrm{~m}^{2} \mathrm{~s}^{-1}$ for DPC bound form. The diffusion coefficients of indole proton of $\operatorname{Trp}^{3}$ appeared in the $10-11 \mathrm{ppm}$ region are $4.67 \times 10^{-10} \mathrm{~m}^{2} \mathrm{~s}^{-1}$ for free peptide and $7.80 \times 10^{-11} \mathrm{~m}^{2} \mathrm{~s}^{-1}$ for DPC micelles bound peptide, respectively. Large changes in diffusion coefficients of indole proton as well as amide proton of $\operatorname{Trp}^{3}$ upon binding to DPC micelle were observed compared to other NH protons. It implies that $\operatorname{Trp}^{3}$ plays important roles in interaction with phospholipid membrane.

In conclusion, the bactericidal action of 9Pbw2 is not due to the formation of transmembrane channels nor to the perturbation of the cell membrane. Trp residues are known to have important roles in the interactions between peptides and biological membranes. ${ }^{19,20}$ The partial insertion of 
hydrophobic residues including Trp residue in the middle portion play important roles in the interactions between peptide and biological membrane. Also, three positively charged residues from $\mathrm{Lys}^{7}$ to $\mathrm{Arg}^{9}$ at the C-terminus in 9Pbw2 may be important for the primary binding to the negatively charge phospholipid head groups in bacterial cell membranes and critical factor for their intracellular mechanism.

\section{Acknowledgments}

This work was supported by Konkuk University in 2011.

\section{REFERENCES}

1. R. E. W. Hancock, and H. G. Sahl, Nat. Biotechnol. 24, 1551. (2006).

2. R. M. Epand, and H. J. Vogel, Biochim. Biophys. Acta 1462, 11. (1999).

3. S. Chilukuri, FEMS Microbiology Letters 160, 91. (1998).

4. R. E. Hancock, and A. Rozek, FEMS Microbiol. Lett. 206, 143. (2002).

5. J. S. Hwang, B. R. Kang, S. R. Kim, E. Y. Yun, K. H. Park, J. P. Jeon, S. H. Nam, H. J. Suh, M. Y. Hong, and I. Kim, Int. J. Indust. Entomol. 17, 131. (2008).

6. S. Shin, J. K. Kim, J. Y. Lee, K.W. Jung, J. S. Hwang, J. Lee, D. G. Lee, I. Kim, S. Y. Shin, and Y. Kim, J. Pept. Sci. 15, 559. (2009).

7. A. Derome, and M. Willamson, J. Magn. Reson. 88, 177. (1990).

8. A. Bax, and D. G. Davis, J. Magn. Reson. 65, 355. (1985).

9. S. Macura, and R.R. Ernst, Mol. Phys. 41, 95. (1980).

10. A. Bax, and D. G. Davis, J. Magn. Reson. 63, 207. (1985).

11. G. Bodenhausen, and D. J. Ruben, J. Chem. Phys. Lett. 69, 185. (1980).

12. A. T. Brünger, X-PLOR Manual, Version 3.1, Yale University, New Haven, CT (1993). 
13. G. M. Clore, and A. M. Gronenborn, CRC. Rev. in Biochem. Biol. 24, 479. (1989).

14. G. M. Clore, and A. M. Gronenborn, Protein Science 3, 372. (1994).

15. K. Wüthrich, M. Billeter, and W. Braun, J. Mol. Biol. 169, 949. (1983).

16. G. M. Clore, A. M. Gronenborn, M. Nilges, and C. A. Ryan, Biochemistry 26, 8012. (1987).

17. M. Nilges, G. M. Clore, and A. M. Gronenborn, FEBS Lett. 229, 317. (1988).

18. K. F. Morris, and C. S. Johnson, J. Am. Chem.114, 3139. (1992).

19. A. Chattopadhyay, S. S. Rawat, D. V. Greathouse, D. A. Kelkar, and R. E. Koeppe, Biophys. J. 95, 166. (2008).

20. R. Domanov, Y. Pietiäinen, V. P. Kontinen, and P. K. Kinnunen, Biochim Biophys Acta 1778, 983. (2008). 